\title{
CRITICAL POINT THEORY FOR NONLINEAR EIGENVALUE PROBLEMS WITH INDEFINITE PRINCIPAL PART
}

\author{
BY
}

\author{
ME LVYN S. BERGER( ${ }^{(1)}$
}

\begin{abstract}
A study of the nontrivial solutions of the operator equation $L u=\lambda r^{\prime}(u)$ is made, where $L$ is a selfadjoint Fredholm operat or mapping a Hilbert space $H$ into itself, and $r(u)$ is a $C^{\prime}$ weakly sequentially continuous real-valued functional defined on $H$. Applications are given to the theory of semilinear elliptic boundary value problems and periodic solutions of Hamiltonian systems.
\end{abstract}

In this article we study the nontrivial solutions of the nonlinear operator equation

$$
L u=\lambda \tau^{\prime}(u) \quad(\lambda \neq 0)
$$

where $L$ is a bounded selfadjoint operator with closed range mapping a Hilbert space $H$ into istelf such that $\operatorname{dim} \operatorname{Ker} L<\infty$, and $\pi(u)$ is a $C^{1}$ functional defined on $H$ with Fréchet derivative $\gamma^{\prime}(u)$ and $\pi^{\prime}(0)=0$. Equations of this type arise naturally in the study of periodic solutions of Hamiltonian systems [1], as well as in the study of the solutions of boundary value problems for semilinear elliptic partial differential equations [2]. The method that has proven successful in each of the above references is based on isoperimetric variational problems in the calculus of variations. Here we show that these examples can be unified in a Hilbert space context by straightforward methods of critical point theory.

A particular difficulty with the study of the solutions of $(0.1)$ is the fact that $\operatorname{Ker} L$ is generally nontrivial while the quadratic form $(L u, u)$ may be indefinite. In either case, a nontrivial solution of $(0.1)$ will not correspond to either an absolute minimum of the functional $I(u)=1 / 2(L u, u)-\lambda \pi(u)$ (which will generally be $-\infty)$ or (for similar reasons) to an extremum of $\pi(u)$ with the constraint $(L u, u)=$ const. Thus, from the point of view of the calculus of variations, any nontrivial solution of $(0.1)$ will be a true critical point of "minimax type". Such critical points are generally treated by topological methods. How-

Received by the editors April 25, 1972 and, in revised form, February 6, 1973. AMS (MOS) subject classifications (1970). Primary 58E 05, 58E15, 35 J60.

Key words and phrases. Nonlinear eigenvalue problems, nonlinear operator equation, semilinear elliptic boundary value problem.

(1) Research partially supported by National Science Foundation Grant GP-36418X and by an AFOSR Grant 73-2437. 
ever an important part of our work is the demonstration that such critical points often can be treated analytically by generalizing the notion of orthogonality to a nonlinear context. This generalization is based on a "principle of natural constraints" which we discuss in \$I.

For example, in global differential geometry, a simple closed geodesic on a compact Riemannian manifold $(M, g)$ is called nontrivial if it is not a point of $\pi$. Clearly closed nontrivial geodesics are thus minimax points of the arclength functional. Currently such closed geodesics are studied by deep topological methods. However, in [6], Poincaré showed in the case of two-dimensional ovaloids that a simple closed geodesic $\gamma$ may be found as a minimum of the arclength functional subject to the constraint that the "integra curvatura" of $M$ is bisected by $\gamma$ (i.e., $\gamma$ divides $M$ in two pieces $M_{1}$ and $M_{2}$ and on each the integra curvatura is $2 \pi$ ). The Gauss-Bonnet theorem, then, implies that this constraint is a "natural" one in the sense that it is satisfied by every nontrivial simple closed geodesic.

The basic idea behind this "principle of natural constraints" is that a critical point of minimax type of a functional $I(u)$ defined on $H$ can be reduced to a study of the absolute minimum of $I(u)$ restricted to an appropriate submanifold defined by the natural constraints. However, in order to solve the resulting isoperimetric problem in the Hilbert space $H$, certain compactness restrictions must be placed on the operator $\gamma^{\prime}(u)$. These compactness restrictions are easily expressed in terms of the functional $\Re(u)$ by saying that $\Re(u)$ is sequentially weakly continuous (i.e., whenever $u_{n} \rightarrow u$ weakly in $H, r\left(u_{n}\right) \rightarrow \Re(u)$ ).

It is important to distinguish this sequential notion from continuity with respect to the weak topology in $H$. Indeed in almost all the cases we consider, the functional $\Re(u)$ will not be continuous with respect to the weak topology in $H$. The notion of weak sequential continuity is, therefore, used here to specify the continuity properties of $\Re(u)$ relative to weak convergence in $H$, substituting for the weak continuity property.

The author is grateful to the referee for a number of helpful suggestions.

The organization of our work is as follows. In $\$ \mathrm{I}$, we mention the basic preliminaries concerning critical point theory in Hilbert space which will be used in the following sections. In $\S \mathrm{II}$, we treat the problem of finding one-parameter families of solutions of $(0.1)$ by global methods without regard to norm. $\$$ III is concerned with local results strengthening those of $\$ \mathrm{II}$, provided one has a first approximation to the nontrivial solution of $(0.1)$. $\$ \mathrm{IV}$ is given over to applications of the results of the previous sections to the study of solutions of various differential systems.

I. Preliminaries. We begin by considering the nontrivial solutions of $(0.1)$ from the point of view of critical point theory. By nontrivial solutions of $(0.1)$ we 
mean those pairs $(u, \lambda) \in H \times \mathbf{R}^{1}$ which satisfy $(0.1)$ and neither $u$ nor $\lambda$ is zero. As we mentioned in the introduction, if $\operatorname{Ker} L \neq\{0\}$, such solutions are not readily obtained by standard critical point theory. If the operator $\gamma^{\prime}(u)$ were linear, however, one could consider the equation ( 0.1$)$ on the orthogonal complement of $\operatorname{Ker} L,[\operatorname{Ker} L]^{\perp}$ in $H$. Moreover, on $[\operatorname{Ker} L]^{\perp}$ standard methods apply. Since the operator $\gamma^{\prime}(u)$ considered here is nonlinear, we shall find a nonlinear analogue by substituting a (curved) manifold $\pi$ in $H$ for the linear subspace $[\operatorname{Ker} L]^{\perp}$. In order to find such a manifold $M$ we define the notion of a natural constraint $\mathcal{S}=\{u \mid f(u)=0\}$ for $(0.1)$ as follows.

Definition. $S=\{u \mid f(u)=0\}$ is a natural constraint for $(0.1)$, if

(i) all nontrivial solutions of $(0.1)$ are in the set $\mathcal{S}$, and

(ii) the real-valued function $f$ is $C^{1}$ on $H$.

In the present case since $[\operatorname{Ker} L]^{\perp}=\{u \mid u \in H,(u, w)=.0, w \in \operatorname{Ker} L\}$, by choosing an orthonormal basis for $\operatorname{Ker} L,\left[w_{1}, \cdots, w_{N}\right]$, the sets $\mathcal{S}_{i}=\left\{u \mid \sigma^{\prime}(u), w_{i}\right)=$ 0\} $(i=1, \cdots, N)$ are natural constraints for $(0.1)$ provided $\gamma$ is $C^{2}$. Indeed, if $u$ is a nontrivial solution of $(0.1)$, taking the inner product of $(0.1)$ with $w_{i} \epsilon$ $\operatorname{Ker} L$, we find $\left.O \tau^{\prime}(u), w_{i}\right)=0$ by virtue of the selfadjointness of $L$. In our work of $\S \mathrm{II}, \bigcup_{i=1}^{N} \delta_{i}$ will be the desired analogue of $[\operatorname{Ker} L]^{\perp}$.

Thus in order to solve $(0.1)$ we shall reformulate the solutions of $(0.1)$ as the extrema of a real-valued function $G_{0}(u)$ defined on some contsraint set $C$, defined by a finite number of $C^{1}$ real-valued functions $G_{i}(u)(i=1, \ldots, N)$ defined on $H$. In order to determine the operator equation satisfied by such a critical point, we prove

Lemma A. Suppose $u_{0}$ is an extremum of the $C^{1}$ functional $G_{0}(u)$ subject to the constraint $C=\left\{u \mid G_{i}(u)=c_{i}(i=1, \cdots, N)\right\}$, where the numbers $c_{i}$ are constants. Then there are numbers $\lambda_{i}$ (not all zero) such that

$$
\sum_{i=0}^{N} \lambda_{i} G_{i}^{\prime}\left(u_{0}\right)=0
$$

where $G_{i}^{\prime}\left(u_{0}\right)$ denotes the Fréchet derivative of $G_{i}(x)$ at $u_{0}$.

Proof. We argue by contradiction, by assuming that the vectors $G_{i}^{\prime}\left(u_{0}\right)$ $(i=0, \cdots, N)$ are linearly independent. Let the extreme value of $G_{0}(u)$ on $C$ be $c_{0}$, then we show that if $(1.1)$ is never satisfied, we can find a curve $u(t) \epsilon$ $C$ for $|t|$ sufficiently small with $u(0)=u_{0}$ such that $G_{0}(u(t))=c_{0}+t$. Since $t$ can be positive or negative, this contradicts the fact that $u_{0}$ is an extremum for $G_{0}$ on $C$. To this end, let $u(t)=u_{0}+\sum_{j=0}^{N} a_{j}(t) w_{j}$ where the real-valued functions $a_{j}(t)$ and the vectors $w_{j}$ are to be determined such that 


$$
a_{j}(0)=0, \quad G_{0}(u(t))=c_{0}+t, \quad G_{i}(u(t))=c_{i} \quad(i=1, \ldots, N) .
$$

Assuming the $w_{j}$ are given, we can find the functions $a_{j}(t)$ satisfying (1.2) provided that we can solve the initial value problem

$$
\left\{\begin{array}{l}
\frac{d}{d t} G_{i}\left[u_{0}+\sum_{j=0}^{N} a_{j}(t) w_{j}\right]=\gamma_{i} \quad(i=0, \ldots, N) \\
a_{j}(0)=0
\end{array}\right.
$$

where $\gamma_{0}=1$ and $\gamma_{i}=0$ for $i>0$. Simplifying (1.3) we can rewrite this initial solution to the problem in the vector form

$$
\mathscr{R}(a(t)) d a / d t=\gamma, \quad a(0)=0,
$$

where $a(t)=\left(a_{0}(t), \ldots, a_{N}(t)\right), \gamma=(1,0, \ldots, 0)$, and $\mathscr{R}\left(a(t)=\left(a_{i j}\right)\right)$ is the $(N+1) \times(N+1)$ matrix with entries $a_{i j}=\left(G_{i}^{\prime}\left(u_{0}+a(t) \cdot w\right), w_{j}\right)$ with $w=$ $\left(w_{0}, w_{1}, \cdots, w_{N}\right)$. Now by the existence theorem for systems of ordinary differential equations, (1.4) and consequently (1.2) has solutions provided the matrix $\mathscr{U}(a(t))$ has an inverse, for $|t|$ sufficiently small, that depends continuously on $a(t)$. Clearly this will be the case provided $\operatorname{det}|\operatorname{R}(a(0))| \neq 0$. Thus we shall choose vectors $w_{j}$ such that the determinant $D$, whose entries are $\left(G_{i}^{\prime}\left(u_{0}\right), w_{j}\right)$ $(i, j=0, \ldots, N)$, is different from zero. To this end we shall make use of the fact that the vectors $G_{i}^{\prime}\left(u_{0}\right)(i=0, \ldots, N)$ are linearly independent, by assumption.

In fact with $w_{j}=G_{j}^{\prime}\left(u_{0}\right)$, suppose $D=\operatorname{det}\left|G_{i}^{\prime}\left(u_{0}\right), G_{j}^{\prime}\left(u_{0}\right)\right|=0$. Then the system of linear equations

$$
\sum_{j} \beta_{j}\left(G_{i}^{\prime}\left(u_{0}\right), G_{j}^{\prime}\left(u_{0}\right)\right)=0 \quad(i=0, \ldots, N)
$$

has a nontrivial solution, $\beta_{j}$ (say). On the other hand, multiplying the equation (1.5) by $\beta_{i}$ and summing we find that for $\beta_{i}=\bar{\beta}_{i}$ (say),

$$
\left\|\sum_{i=0}^{N} \bar{\beta}_{i} G_{i}^{\prime}\left(u_{0}\right)\right\|=0 \quad \text { which implies } \sum_{i=0}^{N} \bar{\beta}_{i} G_{i}^{\prime}\left(u_{0}\right)=0 .
$$

Since the vectors $G_{i}^{\prime}\left(u_{0}\right)$ are linearly independent, (1.6) shows that $\bar{\beta}_{i}=0$ $(i=0, \cdots, N)$. This however contradicts the fact that $D=\operatorname{det}\left|\left(G_{i}^{\prime}\left(u_{0}\right), G_{j}^{\prime}\left(u_{0}\right)\right)\right|=$ 0 . Thus $D \neq 0$ and the curve $u(t) \in C$ exists for $|t|$ sufficiently small, and therefore $G_{0}(u(t))=c_{0}+t$.

On the other hand, in order to solve (0.1) locally, as we shall do in $\S \mathrm{III}$, we 
shall write $H$ as a direct sum $H=\operatorname{Ker} L \oplus H_{0}$ where $H_{0}=[\operatorname{Ker} L]^{\perp}$. Let $P_{H_{0}}$ be the projection of $H \rightarrow H_{0}$, then $P_{H_{0}} L=L$ on $H_{0}$ and for $\lambda \neq 0$ the equation (0.1) can be written as the system

$$
\begin{aligned}
& L w=\lambda P_{H_{0}} \Re^{\prime}(v+w), \\
& \left(I-P_{H_{0}}\right) \Re^{\prime}(v+w)=0
\end{aligned}
$$

where $v \in \operatorname{Ker} L$ and $w \in H_{0}$. Now the question arises as to whether critical point theory can be applied to the system (1.7)-(1.8). In this connection, we prove

Lemma B. Suppose (1.8) uniquely determines $v$ as a function of $w$, say $\boldsymbol{v}=\boldsymbol{g}(w)$ (with $g$ differentiable). Then the solutions of (1.7) coincide with the critical points of the functional $g(w)=1 / 2(L w, w)-\lambda \Re(w+g(w))$ on $H_{0}$.

Proof. First we compute $(d / d \epsilon))\left.\tau(w+\epsilon v+g(w+\epsilon v))\right|_{\epsilon=0}$ for $v \in H_{0}$. Now

$$
(d / d \epsilon) \Re\left(\left.(w+\epsilon v+g(w+\epsilon v))\right|_{\epsilon=0}=\left(\Re(w+g(w)), v+g^{\prime}(w) v\right) .\right.
$$

Since $g(w): H_{0} \rightarrow \operatorname{Ker} L$ and is differentiable, $g^{\prime}(w): H_{0} \rightarrow \operatorname{Ker} L$. Thus $g^{\prime}(w) v$ $=0$ and the right-hand side of (1.9) becomes $O((w+g(w)), v)$. Consequently the Gateaux derivative of $g(w)$

$$
\begin{aligned}
& \left(g^{\prime}(w), v\right)=\left(L w-\lambda \pi^{\prime}(w+g(w)), v\right) \quad \text { for } v \in H_{0} \text {, } \\
& =\left(L w-\lambda r^{\prime}(w+g(w)), P_{H_{0}} u\right) \text { if } v=P_{H_{0}} u, \\
& =\left(L w-P_{H_{0}} \lambda r^{\prime}(w+g(w)), u\right) \text { for all } u \in H \text {. }
\end{aligned}
$$

Consequently $L w=\lambda P_{H_{0}} \gamma^{\prime}(w+g(w))$. The converse statement follows from reversing the augument just given.

Another result of importance in the study of the solutions of $(0.1)$ consists in establishing that the system of natural constraints for $(0.1)$ is nonvacuous. For systems of the form $L u=\lambda \gamma^{\prime}(u)$, a set of natural constraints obtained above were

$$
\delta_{i}=\left\{u \mid\left(\Re^{\prime}(u), w_{i}\right)=0\right\} \quad(i=1, \cdots, N) .
$$

These constraints can be written

$$
\left(r^{\prime}\left(v+\sum_{i=1}^{N} \beta_{i} w_{i}\right), w_{j}\right)=0 \quad(j=1, \ldots, N) .
$$

Regarding (1.11) as a function of $\beta=\left(\beta_{1}, \ldots, \beta_{N}\right)$ and fixing $v \in[\operatorname{Ker} L]^{\perp}$, 
(1.10) is satisfied by those $N$-vectors $\beta$ that are critical points of the functional $F(\beta)=r\left(v+\sum_{i=1}^{N} \beta_{i} w_{i}\right)$. Now we prove

Lemma C. Suppose $\Re(u)$ is a $C^{1}$ strictly convex function of $u$ witb $\Re(u) \geq$ $\gamma(0)=0$; then the constraint set

$$
C_{R}=\left\{u \mid r(u)=R,\left(\Re^{\prime}(u), w\right)=0, w \in \operatorname{Ker} L\right\}
$$

is nonvacuous for eacb $R>0$.

Proof. First we note that by virtue of the remarks just above and the strict convexity of the functional $\pi$, there is one and only one critical point $\beta(v)=$ $\left(\beta_{1}(v), \cdots, \beta_{N}(v)\right)$ of $F(\beta)=\pi\left(v+\sum_{i=1}^{N} \beta_{i} w_{i}\right)$ for fixed $v \in[\operatorname{Ker} L]^{\perp}$. Thus for each positive $s$, there is an element $v(s)=s v+\sum_{i=1}^{N} \beta_{i}(s) w_{i}$ such that $\left.\sigma^{\prime}(v(s)), w\right)=0$ for all $w \in \operatorname{Ker} L$. Now for fixed nonzero $v \in[\operatorname{Ker} L]^{\perp}$ the function $G\left(s, \beta_{1}, \cdots, \beta_{N}\right)=\pi\left(s v+\sum_{i=1}^{N} \beta_{i} w_{i}\right)$ is a strictly convex function defined on $\mathbf{R}^{N+1}$, so that as $|s|+\sum_{i=1}^{N}\left|\beta_{i}\right| \rightarrow \infty, G\left(s, \beta_{1}, \cdots, \beta_{N}\right) \rightarrow \infty$. Hence, as a function of $s, g(s)=\Re\left(s v+\sum_{i=1}^{N} \beta_{i}(s v) w_{i}\right) \rightarrow \infty$ as $s \rightarrow \infty$. Consequently the lemma is proved provided we show that the function $g(s)$ is a continuous function of $s$. This is immediate from convexity theory (see [3, pp. 255-256]) since $g(s)=\inf G\left(s, \beta_{1}, \cdots, \beta_{N}\right)$ over $\beta_{1}, \cdots, \beta_{N}$ and $G$ is strictly convex.

Remark. If $\Re(0) \neq 0$, an analogue of Lemma $C$ clearly holds provided $R$ is chosen sufficiently large. Similarly if $\Re(u)$ is strictly convex only for $|u|$ sufficiently large, Lemma $C$ holds provided again $R$ is also chosen sufficiently large.

II. Some global results. We are now in a position to prove

Theorem 1. Suppose $L$ is a bounded selfadjoint Fredbolm operator mapping a real Hilbert space $H$ into itself, and sucb that $(L u, u) \geq 0$. Suppose $\Re(u)$ is a $\mathbf{C}^{2}$ strictly convex functional defined on $H$ that is weakly sequentially continuous and sucb that $\Re(u) \geq \Re(0)=0$. Set $C_{R}=\left\{u \mid u \in H, \Re(u)=R, O{ }^{\prime}(u), w\right)=0 \forall w \in$ Ker $L\}$. Then provided

$$
(L u, u) \rightarrow \infty \text { as }\|u\| \rightarrow \infty \text { for } u \in C_{R},
$$

the equation $L u=\lambda \gamma^{\prime}(u)$ bas a one parameter family of nontrivial solutions $(u(R), \lambda(R))$ with $\gamma(u(R))=R$ and $\lambda(R)>0$. Furthermore $u(R)$ is characterized as an element in $C_{R}$, that attains inf $(L u, u)$ for $u \in C_{R^{*}}$.

Proof. First we show that any element $u_{0}$ that achieves the minimum of $G_{0}(u)=1 / 2(L u, u)$ subject to the constraints $C_{R}=\left\{u \mid u \in H, \gamma(u)=R, O{ }^{\prime}(u), w\right)=$ 0 for all $w \in \operatorname{Ker} L\}$ is a solution of the equation $L u=\lambda r^{\prime}(u)$ which obviously has the property that $\Re\left(u_{0}\right)=R$. Indeed by Lemma $A, u_{0}$ must satisfy an equation of the form 


$$
\beta_{0} L u=\beta_{1} r^{\prime}(u)+\sum_{i=2}^{N} \beta_{i} r^{\prime \prime}(u) w_{i}
$$

where $\left(w_{1}, \cdots, w_{N}\right)$ denotes an orthonormal basis for $\operatorname{Ker} L$ and $\beta_{i}$ $(i=0,1, \cdots, N)$ are constants. We show that the constants $\beta_{i}=0$ for $i>1$ while $\beta_{0}$ and $\beta_{1}$ are not zero. Taking the inner product of (2.1) with $\beta_{j} w_{j}$ and using the facts that $L w_{j}=0$ and $\left.O \tau^{\prime}(u), w\right)=0$ for any $w \in \operatorname{Ker} L$, we find that, for $\left.\bar{w}=\sum_{i=1}^{N} \beta_{i} w_{i}, O^{\prime \prime}(u) w, w\right)=0$. Since $r^{\prime \prime}(u)$ is selfadjoint, $\gamma^{\prime \prime}(u) \bar{w}=$ 0. Since $\pi$ is strictly convex, $\operatorname{Ker} r^{\prime \prime}(u)=0$, so that $\bar{w}=0$, i.e., $\beta_{i}=0$, $i>1$. Now $\beta_{0} \neq 0$, since if it were zero, $\beta_{1} \neq 0$ so that $\Re^{\prime}(u)=0$. In this case $u=0$, since $\pi$ is strictly convex, which contradicts the fact that $r(u)=R>0$. To demonstrate that $\beta_{1} \neq 0$, we suppose the contrary, then $L u_{0}=0$ so $u_{0} \epsilon$ Ker $L$. Thus since $u_{0} \in C_{R^{\prime}}$ Or' $\left.\left(u_{0}\right), u_{0}\right)=0$; and again by the strict convexity of $\Re(u), u_{0}=0$ contradicting again the fact that $\gamma\left(u_{0}\right)=R>0$.

Next we show that inf $1 / 2(L u, u)$ over $C_{R}$ is attained by an element $\bar{u} \in C_{R}$. By hypothesis, $(L u, u) \geq 0$ and by virtue of Lemma $C$, the constraint set $C_{R}$ is nonempty for each $R>0$. Thus denoting inf $1 / 2(L u, u)$ over $C_{R}$ by $\alpha$, we suppose we have a sequence $u_{n} \in C_{R}$ such that $1 / 2\left(L u_{n}, u_{n}\right) \leq a+1$ and $1 / 2\left(L u_{n}, u_{n}\right)$ $\rightarrow$ a. Letting $u_{n}=v_{n}+w_{n}, v_{n} \in \operatorname{Ker} L$ and $w_{n} \in H_{0}=[\operatorname{Ker} L]^{\perp}$, we find $\left(L w_{n}, w_{n}\right) \leq a+1$. Since $L$ has closed range, there is a constant $c>0$ independent of $n$ such that $\left(L w_{n}, w_{n}\right) \geq c\left\|w_{n}\right\|^{2}$. Consequently, $\left\|w_{n}\right\|$ is uniformly bounded. Now we show that $\left\|v_{n}\right\|$ is bounded uniformly. If $\left\|v_{n}\right\| \rightarrow \infty$ while $\left\|w_{n}\right\| \leq$ const, by the coerciveness hypothesis of the theorem, $u_{n}=v_{n}+w_{n} \in \partial C_{R}$, implies that $\left(L u_{n}, u_{n}\right) \rightarrow \infty$ which contradicts the fact that $1 / 2\left(L u_{n}, u_{n}\right) \leq \alpha+1$. Thus $u_{n}=v_{n}+w_{n}$ is a bounded sequence in $H$, and consequently has a weakly convergent subsequence $w$ ith weak limit $\bar{u}$ (say). Clearly $(L u, u)$ is lower semicontinuous with respect to weak convergence, so that $1 / 2(L \bar{u}, \bar{u}) \leq \alpha$. Now we prove that $1 / 2(L \bar{u} u \bar{u})=a$ by showing that $C_{R}$ is weakly closed, since then $\bar{u} \epsilon$ $C_{R}$. Now the functional $\Re(u)$ is continuous with respect to weak convergence, so that if $u_{n} \rightarrow u$ weakly in $H$ with $u_{n} \in C_{R}, \Re\left(u_{n}\right)=\Re(u)=R$. Similarly since $r$ is a $C^{2}$ functional continuous with respect to weak convergence, $r^{\prime}(u)$ maps weakly convergent sequences into strongly convergent sequences. Thus $0=$ $\left.\left.O^{\prime}\left(u_{n}\right), w\right)=O \tau^{\prime}(u), w\right)$ for all $w \in \operatorname{Ker} L$. Thus $C_{R}$ is weakly closed, and the theorem is proved.

In order to handle the case in which the operator $L$ has negative eigenvalues, we prove the following

Theorem 2. Theorem 1 bolds without the bypotbesis that $(L u, u) \geq 0$, provided we suppose the essential spectrum of $L \subset(0, \infty)$ (i.e., the set $\{u\}(L u, u) \leq 0\}$ is finite dimensional). In this case $\lambda(R)$ may be negative. 
Proof. By repeating the first patt of the proof of Theorem 1, we note that it suffices to show that $\inf 1 / 2(L u, u)$ over $C_{R}$ is attained by an element $u_{0} \in C_{R}$. Since $L$ has closed range, finite dimensional kernel, and is a bounded selfadjoint operator, $L$ can be written as the difference of two selfadjoint nonnegative operators $L_{1}$ and $L_{2} ; L=L_{1}-L_{2}$. In fact, $L_{2}$ is compact, since the essential spectrum of $L$ is nonnegative. Consequently the quadratic form $(L u, u)$ is lower semicontinuous with respect to weak convergence in $H$. Thus to show that the infimum of $(L u, u)$ over $C_{R}$ is attained, it remains to prove:

(i) $(L u, u)$ is bounded below by a finite number $a$ (say) on $C_{R^{\prime}}$,

(ii) the set $\left\{u \mid u \in C_{R} a \leq(L u, u) \leq a+1\right\}$ is uniformly bounded in $H$.

Now the fact that the $L_{2}$ is compact implies that negative spectrum of $L$ is bounded below and consequently $(L u, u)$ can tend to $-\infty$ only if $\|u\| \rightarrow \infty$. Thus (i) must hold, since by the coerciveness hypothesis, $(*)$ of the theorem implies that $(L u, u) \rightarrow+\infty$ is $\|u\| \rightarrow \infty$ for $u \in C_{R}$. Similarly, this coerciveness hypothesis (*) also yields (ii). Thus Theorem 2 is established.

Furthermore the smoothness restriction on $\gamma^{\prime}(u)$ may be reduced by virtue of the following

Corollary A. Theorems 1 and 2 continue to bold if the smoothness requirement on $\pi(u)$ is reduced to $C^{1}$. However, in this case, the extremal characterization of the pair $(u(R), \lambda(R))$ may no longer bold.

Proof. First we observe that the $C^{2}$ hypothesis on $\pi(u)$ was needed only in case $\operatorname{Ker} L$ was nontrivial. Thus we shall apply Theorems 1 and 2 to an ammended operator equation on the subspace $H^{1}=[\operatorname{Ker} L]^{\perp}$ where the operator $L$ is invertible (so that $\mathcal{T}^{\prime}(u)$ need only be $C^{1}$ ). The strict convexity of $\mathcal{T}^{\prime}(u)$ is then used to equate the solutions of original and amended operator equations.

Indeed let $P$ denote the canonical projection of $H$ onto $[\operatorname{Ker} L]^{\perp}$ then the equation $L u=\lambda \tau^{\prime} u$ can be written as the pair

$$
\begin{aligned}
L v & =\lambda \operatorname{Pr}^{\prime}(v+w), & & v \in[\operatorname{Ker} L]^{\perp}, \\
0 & =(I-P) \mathcal{I}^{\prime}(v+w), & & w \in \operatorname{Ker} L .
\end{aligned}
$$

For fixed $v$, the equation (2.2)b has the unique solution $w=g(v)$, by virtue of the strict convexity of $\Re(u)$. In addition, by $[3]$ the strict convexity of $\Re(u)$ and the fact $g(v)$ is characterized the minimum of $\Re(v+w)$ for $w \in \operatorname{Ker} L$ implies that $g(v)$ is differentiable. Consequently by Lemma B of $\$ 1$, the operator equation (2.2)a with $w$ teplaced by $g(v)$ satisfies the hypotheses of Theorems 1 and 2 on $H^{1}=(\operatorname{Ket} L)^{\perp}$ except that the functional $)(v+g(v))$ is now only $C^{1}$. However, on $H^{1}$, Ker $L$ is trivial and so the arguments of Theorem 1 apply directly to yield the desired result. 
The arguments given in Theorems 1 and 2 enable us to easily prove the following necessary and sufficient condition for the existence of nontrivial solutions of (0.1).

Corollary B. Suppose $L$ is a selfadjoint bounded Fredbolm operator mapping $H$ into $H$ with nonnegative essential spectrum. If $r$ is a $C^{2}$ weakly sequentially continuous functional defined on $H$ such that $(\dagger)$ either $\left.O \tau^{\prime}(u), u\right)=0$ or $\gamma(u)=0$ implies $u=0$, and $\gamma^{\prime \prime}(u)$ is injective on $\operatorname{Ker} L$ for $u \neq 0$, then a necessary and sufficient condition for the existence of nontrivial solutions of $(0.1)$ is that the set

$$
C_{R}=\{u \mid u \in H, \Re(u)=R,(\Re(u), w)=0 \text { for } w \in \operatorname{Ker} L\}
$$

is nonempty for some $\dot{R} \neq 0$, provided $(L u, u) \rightarrow \infty$ for $u \in C_{R}$ as $\|u\| \rightarrow \infty$. If $\operatorname{Ker} L=\varnothing$, condition ( $\dagger$ may be replaced by that. $\pi^{\prime}(u) \neq 0$ for $u \in C_{R^{\prime}}$.

Proof. The sufficiency follows by repeating the proof of Theorem 1. The necessity comes from the facts that (a) $\left.\mathcal{O}^{\prime}(u), w\right)=0$ for $w \in \operatorname{Ker} L$ is a natural constraint for $(0.1)$ and $(b)$ if a solution $v$ of $(0.1)$ is not zero, $r(v) \neq 0$. The last statement of the theorem is immediate by virtue of the proof given for Theorem 1.

Remarks. 1. Pohozaev [7] has shown that, for a large class $C$ of functionals $\tau^{\prime}(u)$, any number $R$ in the range of $\tau^{\prime}(u)$ (apart from a possible set of measure zero) has the property that $\gamma^{\prime}(u) \neq 0$ on the hypersurface $\{u \mid \gamma(u)=R\}$. This generalizes the well-known finite dimensional result of $M$. Morse [8]. Consequently, if $\operatorname{Ker} L=\varnothing$, apart from the continuity restrictions on $\pi \in C$ convexity restrictions need not be placed on $\pi$ (provided the real number $R$ is well chosen).

2. If $\operatorname{Ker} L=\varnothing$ and $\pi^{\prime}(u)$ is homogene ous of degree $p>1, \pi(u)=$ $\left.(1 /(p+1)) \gamma^{\prime}(u), u\right)$. Consequently, assuming $\gamma^{\prime}(u)$ is not identically constant, and weakly sequentially continuous, Corollary $B$ and Remark 1 above show that, for each $R>0$, the equation ( 0.1$)$ has nontrivial solutions as in Theorem 1 , provided $(L u, u) \rightarrow+\infty$ for $u \in C_{R}$.

III. Local results. In this section, we prove two distinct fundamental results of a local nature of the nontrivial solutions of the equation $L u=\lambda \gamma^{\prime}(u)$. First we specialize the family of nontrivial solutions $(u(R), \lambda(R))$ found in Theorem 1 by letting $R \rightarrow 0$ and showing that $(u(R), \lambda(R)) \rightarrow\left(0, \lambda_{0}\right)$ where $\lambda_{j}$ is the smallest nonzero positive eigenvalue of the linear equation

$$
L u=\lambda \tau^{\prime \prime}(0) u \text {. }
$$

Secondly we completely remove the convexity restrictions of Theorems 1 and 2 by showing that for every isolated eigenvalue $\lambda_{i}$ of (3.1) of finite multiplicity, 
there is a family of nontrivial solutions $\left(u_{i}(R), \lambda ;(R)\right)$ of equation $(0.1)$ [with $R$ sufficiently small near $\left.\left(0, \lambda_{i}\right)\right]$.

Here we suppose that

$$
r^{\prime}(u)=\tau^{\prime \prime}(0) u+P(u) \text { near } u=0
$$

where $\|P(u)-P(v)\| \leq k(\|u\|,\|v\|)\|u-v\|$ for $\|u\|,\|v\|$ sufficiently small and $k(x, y) \rightarrow 0$ as $|x|+|y| \rightarrow 0$. We begin by proving the first result just mentioned.

Theorem 3. Suppose the operator $L$ and the functional $\pi$ satisfy the bypotbeses of Theorem 1 above, as well as the condition (3.2). Let $\lambda_{j}$ denote the smallest nonzero positive eigenvalue of (3.1). Then, as $R \rightarrow 0$, the nontrivial family of solutions $(u(R), \lambda(R))$ referred to in Theorem 1 tends to $\left(0, \lambda_{j}\right)$, provided $\pi^{\prime \prime}(0)$ maps Ker $L$ into itself.

Proof. The variational characterization of the family $(u(R), \lambda(R))$ as the $\inf _{C_{R}}(L u, u)$ implies that, as $R \rightarrow 0, u(R) \rightarrow 0$. Indeed we shall prove below that $\inf _{C_{R}}(L u, u) \rightarrow 0$ as $R \rightarrow 0$. Consequently, since any element of $u \in C_{R}$ can be written in the form $u=w+v(w), w \in[\operatorname{Ker} L]^{\perp}$ and $v(w) \in \operatorname{Ker} L$ (with $v(w)$ uniquely determined by $w), w(R) \rightarrow 0$ strongly in $H$ as $R \rightarrow 0$, so that $v(w(R))$ and thus $u(R) \rightarrow 0$ as $R \rightarrow 0$. We shall obtain the desired result then by showing that both $\left\{\lambda(R)-\lambda_{j}\right\}$ and $\inf _{C_{R}}(L u, u) \rightarrow 0$ with $R$. To this end setting $\gamma^{\prime \prime \prime}(0)=L_{1}$ we note that

(i) $\lambda_{0}$ can be characterized as follows: $\lambda_{0}=\inf (1 / 2)(L u, u)$ over the set $\partial \Sigma_{R}=\left\{u \mid 1 / 2\left(L_{1} u, u\right)=R,\left(L_{1} u, w\right)=0\right.$ for $\left.w \in \operatorname{Ker} L\right\}$,

(ii) since the pair $(u(R), \lambda(R))$ satisfies the equation (3.1),

$$
\lambda(R)=(L u(R), u(R)) /\left(\Re^{\prime}(u(R)), u(R)\right) .
$$

Now $(L u(R), u(R))=\inf (L u, u)$ over the set $C_{R}=\left\{u \mid \gamma(u)=R, \sigma^{\prime}(u), w\right)=0$ for $w \in \operatorname{Ker} L\}$, and since $r^{\prime}=L_{1}+P$ for any $u \in C_{R}$ with $R$ sufficiently small,

$$
\begin{aligned}
1 / 2\left(\tau^{\prime}(u), u\right) & =\pi(u)-\int_{0}^{1}\left(\pi^{\prime}(s u)-1 / 2 \pi^{\prime}(u), u\right) d s \\
& =R+\int_{0}^{1}\left(P^{\prime}(s u)-1 / 2 P^{\prime}(u), u\right) d s=R+O\left(\|u\|^{3}\right) .
\end{aligned}
$$

Since $\pi(u)=O\left(\|u\|^{2}\right)$ for $\|u\|$ sufficiently small, $\left.O \tau^{\prime}(u(R)), u(R)\right)=R+o(R)$ as $R \rightarrow 0$. Thus as $R \rightarrow 0$, by (3.3),

$$
\lambda_{0}-\lambda(R)=\frac{1}{2 R}\left\{\inf _{\partial \Sigma_{R}}(L u, u)-\inf _{C_{R}}(L u, u)\right\}+o(1) .
$$

To estimate the first term on the right, we note that, by virtue of the arguments of Lemma $C$, any element $u$ of $C_{R}$ can be uniquely written $u=t v+f(t v)$ where 
$\|v\|=1$ and $v \in[\operatorname{Ker} L]^{\perp}$, whereas $f(t v) \in \operatorname{Ker} L$. Clearly a similar representation holds for $\partial \Sigma_{R}: u=s v+g(s v)$ (say). However in this case the operator $g$ is homogeneous of degree 1. (A fact which we shall use later on.) Thus

$$
\begin{aligned}
\inf _{\partial \Sigma_{R}}(L u, u)-\inf _{C_{R}}(L u, u) \\
\quad=\inf _{v \in[\operatorname{Ker} L]+,\|v\|=1}(L(s v), s v)-\inf _{v \in \operatorname{Ker} L,\|v\|=1}(L(t v), t v) \\
\quad=\left(s^{2}-t^{2}\right) \lambda_{0}
\end{aligned}
$$

where $\lambda_{j}$ is the smallest nonzero eigenvalue of $L$. Thus from (3.4) to prove the result, it suffices to show that $s^{2}-t^{2}=o(R)$ as $R \rightarrow 0$, or equivalently that $s^{2}-t^{2}=o\left(\|u\|^{2}\right)$ as $R \rightarrow 0$. To prove this last fact, assume for the moment that the functions $f$ and $g$, defined above, satisfy the estimates

$$
\|f(u)\|=O(\|u\|), \quad\|f(u)-g(u)\|=O\left(\|u\|^{2}\right) \text { as }\|u\| \rightarrow 0 .
$$

Then if $v_{g}(s)=s v+g(s v) \in C_{R}$ and $v_{f}(t)=t v+f(t v) \in \partial \Sigma_{R}$, and setting $Q(v)=$ $1 / 2\left(L_{1} \nu, v\right)$, we find

$$
\begin{aligned}
Q\left(v_{f}(t)\right)+P\left(v_{f}(t)\right) & =R, \\
Q\left(v_{g}(s)\right) & =R_{0}
\end{aligned}
$$

If we replace $Q\left(v_{f}(t)\right)$ in (3.7) by $Q\left(v_{g}(t)\right)+\left[Q\left(v_{f}(t)\right)-Q\left(v_{g}(t)\right)\right]$, use (3.6) and subtract (3.7) and (3.8), we find that

$$
Q\left(v_{\boldsymbol{g}}(t)\right)-Q\left(v_{\boldsymbol{g}}(s)\right)+o\left(t^{2}\right)+P\left(v_{f}(t)\right)=0 .
$$

By virtue of the homogeneity of the operator $g$ and the fact that, for $u \in \partial \Sigma_{R}$, $\left(L_{1} u, w\right)=0$ (for any $w \in \operatorname{Ker} L$ ),

$$
Q\left(v_{g}(t)\right)-Q\left(v_{B}(s)\right)=\left(t^{2}-s^{2}\right) Q\left(v_{B}(1)\right)
$$

Thus (3.9) implies that, for fixed $v \in[\operatorname{Ker} L]^{\perp}$ with $\|v\|=1$,

$$
\left(t^{2}-s^{2}\right) Q\left(v_{B}(1)\right)+o\left(t^{2}\right)+O\left(t^{3}\right)=0
$$

Since, $L$ maps $\operatorname{Ker} L$ into itself,

$$
\left(Q v_{g}(1)\right)=\left(L_{1}(v+g(v)), v\right)=\left(L_{1} v, v\right) \geq \inf _{\|v\|=1, v_{\perp} \operatorname{Ker} L}\left(L_{1} v, v\right)=\lambda_{0}>0
$$

from (3.10) we obtain $\left(s^{2}-t^{2}\right)=o\left(\|u\|^{2}\right)$, as required. Thus it remains to prove 
Lemma D. The estimates (3.6) bold.

Proof. Now $f(u)$ is a mapping of $[\operatorname{Ker} L]^{\perp} \rightarrow \operatorname{Ker} L$ that satisfies

$$
\left(L_{1}(u+f(u))+P^{\prime}(u+f(u)), w\right)=0
$$

for all $w \in \operatorname{Ker} L$, i.e.

$$
\Pi L_{1}(u+f(u))+\Pi P^{\prime}(u+f(u))=0
$$

where $\Pi$ is the canonical projection of $H \rightarrow \operatorname{Ker} L$. Thus $\Pi L_{1}$ is invertible on Ker $L$, by virtue of the strict convexity of $\pi(u)$. So (3.12) implies

$$
\|f(u)\| \leq \text { const }\left\{\left\|L_{1} u\right\|+O(\|u\|+\|f(u)\|)\right\} \text {. }
$$

Consequently $\|f(u)\|=O(\|u\|)$. To estimate $\|f(u)-g(u)\|$ we recall that $g(u)$ is uniquely defined by the relation

$$
\Pi L_{1}(u+g(u))=0 .
$$

Subtracting (3.12) and (3.13) we find

$$
\Pi L_{1}(f(u)-g(u))+\Pi P^{\prime}(u+f(u))=0 .
$$

Thus by virtue of the invertibility of $\Pi L_{1}$ on $\operatorname{Ker} L$,

$$
\|f(u)-g(u)\| \leq\left\|P^{\prime}(u+f(u))\right\|=O\left(\|u\|^{2}\right) .
$$

Thus the estimates (3.6) are established.

Finally, we show that the result of this last theorem can be substantially improved provided only that a local result is desired, by (i) completely removing the convexity hypothesis on the functional $\pi(u)=1 / 2\left(L_{1} u, u\right)+P(u)$, and (ii) finding multiple one-parameter families of nontrivial solutions of (3.2). In order to state our results succinctly, we define $\lambda_{i}$ as a point of bifurcation of (0.1) with respect to the origin if every open neighborhood of $\left(0, \lambda_{i}\right)$ on $H \times \mathbf{R}^{1}$ contains a point $(u, \lambda)$ distinct from $(0, \lambda)$ that satisfies (3.2). We now prove

Theorem 4. Suppose $L-\lambda_{i} \mathcal{T}^{\prime \prime}(0)$ is a selfadjoint bounded mapping of $H$ into $H$ with closed range, and finite-dimensional kernel, furthermore we suppose that $\boldsymbol{r}^{\prime}(u)$ is a $C^{3}$ real-valued function defined in a neigbborbood of the origin and sucb that $\pi^{\prime}(u)=L_{1} u+P(u)$ where $P(u)$ satisfies the conditions of equation (3.2), and $L_{1}=\mathcal{H}^{\prime \prime}(0)$. Then every eigenvalue $\lambda_{i}$ of (3.1) is a point of bifurcation of (3.2) with respect to the origin provided (a) either that $L_{1}$ is a (1-1) map of Ker $L$ into itself, and (b) $L$ is a bounded selfadjoint mapping of $H$ into $H$ with nonnegative spectrum. 
Proof. In order to proceed we shall use Lemma B, i.e., we write $H=\operatorname{Ker} L$ $\oplus H_{0}$ and write an equation equivalent to (3.2) on $H_{0}$. In order to apply Lemma B, we let $\Pi$ be the canonical projection of $H \rightarrow \operatorname{Ker} L$ and show that for $v \in \operatorname{Ker} L$ given $w \in H_{0}$ sufficiently small, the equation $\left.\Pi\right) \gamma^{\prime}(v+w)=0$ can be uniquely solved for $v$ in terms of $w, v=g(w)$ (say) with $g$ differentiable. Indeed, since $\Re^{\prime}(u)=L_{1} u+P(u),(1.8)$ becomes $\Pi L_{1}(v+w)+\Pi P(v+w)=0$. Since $L_{1}$ is selfadjoint, $\Pi L_{1} w=0$ so that

$$
\Pi L_{1} v+\Pi P(v+w)=0 .
$$

Now on $\operatorname{Ker} L, L_{1}$ is invertible since by hypothesis either the map $L_{1}: \operatorname{Ker} L$ $\rightarrow \operatorname{Ker} L$ is $(1-1)$. Thus the implicit function theorem applied to (3.14) implies that there is a unique $C^{1}$ function $g(v)$ such that $v=g(w)$ satisfies (3.14) and $\|g(w)\|=O\left(\|w\|^{2}\right)$. By virtue of Lemma $\mathrm{B}$, in a small neighborhood of the origin the solutions of (3.2) coincide with the solutions of

$$
L w=\lambda\left\{L_{1} w+\varrho^{\prime}(w)\right\}
$$

on $H_{0}$, where $\varrho(w)=\pi(w+g(w))$. Now on $H_{0}, L w$ is $(1-1)$ and since $L$ is a Fredholm map of index zero, $L$ is invertible on $H_{0}$. Furthermore, since $L$ is positive definite on $H_{0}, L^{-1}$ is also positive definite and selfadjoint, and thus has a square root $L^{-1 / 2}$. Consider the critical points in $H_{0}$ of the functional $1 / 2\left(L_{1}\left(L^{-1 / 2} z\right), z\right)+\varrho_{\left(L^{-1 / 2} z\right)}$ on the sphere $\|z\|^{2}=R$ (for $R$ sufficiently small). They are precisely the solutions of

$$
z=\lambda\left\{L^{-1 / 2} L_{1} L^{-1 / 2} z+L^{-1 / 2} \Theta^{\prime}\left(L^{-1 / 2} z\right)\right\}
$$

or

$$
L^{-1 / 2} z=\lambda\left\{L_{1}\left(L^{-1 / 2} z\right)+\Theta^{\prime}\left(L^{-1 / 2} z\right)\right\}
$$

Setting $w=L^{-1 / 2} z, L w=\lambda\left\{L_{1}(w)+\mathcal{G}^{\prime}(w)\right\}$. Thus the solutions of (3.16) are in (1-1) correspondence with the solutions of (3.15). Now to (3.16) we can apply the following theorem [9] which we state as follows:

An eigenvalue $\lambda_{i}$ of $z=\lambda L_{1} z$ is a point bifurcation for the operator equation $z=\lambda\left\{L_{1} z+P^{\prime}(z)\right\}$ at $z=0$ provided $\left(I-\lambda_{i} L_{1}\right)$ is a Fredholm selfadjoint operator mapping $H \rightarrow H$ and for $\|z\|$ sufficiently small $P(z)$ is a $C^{3}$ functional such that $P^{\prime}(0)=P^{\prime \prime}(0)=0$. Since the nonzero eigenvalues of $z=\lambda L^{-1 / 2} L_{1} L^{-1 / 2} z$ are exactly the nonzero eigenvalues of (3.1), we find that the nonzero eigenvalues of (3.1) are points of bifurcation of (3.2). Thus the theorem is established.

Remark. Actually the provisos of Theorems 3 and 4 can be considerably weakened, by a closer study of bifurcation theory. Indeed we conjecture that the hypothesis (a) and the assumption concerning the spectrum of $L$ in 4 may be removed completely. We hope to carry this extension out in a later publication. 
IV. Applications. Here we apply the results of the previous sections to some specific nonlinear problems in the theory of ordinary and partial differential equations.

(i) The Diricblet problem for semilinear elliptic partial differential equations. Let $\Omega$ be a bounded domain (with smooth boundary) in $R^{N}$, and suppose that a formally selfadjoint elliptic operator $L$ (of order $2 m$ ) defined on $\Omega$ can be written in the form

$$
L u=\sum_{|a|,|\beta| \leq m}(-1)^{|a|} D^{\alpha}\left\{a_{a \beta}(x) D^{\beta} u\right\}
$$

where the coefficients $a_{a \beta}(x)$ are smooth (i.e., of class $C^{a, \mu}$ (say) for some $0<$ $\mu<1)$. Now we consider the existence of nontrivial solutions of the following semilinear elliptic boundary value problem:

$$
\begin{aligned}
& L u=\lambda \sum_{|a|,|\beta| \leq m-1}(-1)^{|a|} D^{a}\left\{f_{a}\left(x, D^{\beta} u\right)\right\} \text { in } \Omega, \\
& \left.D^{a} u\right|_{\partial \Omega}=0, \quad|a| \leq m-1 .
\end{aligned}
$$

As an application of Theorem 2, we prove

Theorem 5. Suppose $f_{\alpha}(x, \xi)=\partial F(x, \xi) / \partial \xi_{a^{\prime}}$ for $|a| \leq m-1$, where $F(x, \xi)$ $=F\left(x, \xi^{\beta}\right),|\beta| \leq m-1$, is a $C^{1}$ strictly convex even function of $\xi$ with $F(x, 0)$ $=\partial F(x, 0) / \partial \xi_{a}=0$ for fixed $x \in \Omega$, and satisfies the following growth conditions (for $|u|+|D u|+\cdots+\left|D^{m} u\right|$ sufficiently large)

$$
F(x, \xi) \rightarrow \infty \text { as }|\xi| \rightarrow \infty \text { uniformly for } x \in \Omega
$$

where $\sigma_{a \gamma}<\sigma_{\gamma}\left\{1-\sigma_{a}^{-1}\right\}$ and for $\delta>0, \sigma_{\delta}=2 N /(N-2(m-\delta))$. Then the system (4.2)-(4.3) bas a nontrivial solution $(u(R), \lambda(R))$ for each $R>0$ with the property that $\int_{\mathbf{Q}} F(x, u(R))=R$.

Proof. The proof can be divided into two parts: (1) the existence of nontrivial weak solutions of (4.2)-(4.3), and (2) the proof of the regularity of the nontrivial weak solutions. Since (2) follows by arguments now standard, using the regularity theory for linear elliptic equations (cf. for example [2, pp. 168-172]), it suffices to prove part (1). Recall that a weak solution of the system (4.2)-(4.3) is an element $u \in \mathfrak{W}_{m, 2}(\Omega)$ which satisfies the following integral identity for all test functions $\eta \in \stackrel{i}{W}_{m, 2}^{m}(\Omega)$, 


$$
\sum_{|a|,|\beta| \leq m} \int_{Q} a_{a \beta}(x) D^{a} u D^{\beta} \eta=\lambda \sum_{|\alpha|,|\beta|_{\leq m}} \int_{\mathbf{Q}} f_{a}\left(x, D^{\beta} u\right) D^{\alpha} \eta_{\bullet}
$$

Furthermore, such weak solutions are in $(1-1)$ correspondence with the solutions of the operator equations in $\mathscr{W}_{m, 2}(\Omega)$ of the form

$$
L u=\lambda f(u)
$$

where the operators $L, f$ mapping $\mathbb{W}_{m, 2}(\Omega)$ into itself are defined implicitly by

$$
\begin{aligned}
& (L u, \eta)=\sum_{|a|,|\beta| \leq m} \int_{Q} a_{a \beta}(x) D^{a} u D^{\beta} \eta, \quad \forall \eta \in C_{0}^{\infty}(\Omega), \\
& (f(u), \eta)=\sum_{|a|,|\beta| \leq m-1} \int_{Q} f_{a}\left(x, D^{\beta} u\right) D^{a} \eta, \quad \forall \eta \in C_{0}^{\infty}(\Omega) .
\end{aligned}
$$

Thus if we can verify the hypotheses of Theorem 1 for the abstract operators $L$ and $N$, we shall have proven our result. By virtue of definition (4.7), it is clear that $L$ is a bounded selfadjoint operator on $W_{m, 2}(\Omega)$. The facts that $L$ has a closed range and finite dimensional kernel follow immediately from Garding's inequality and Rellich's lemma. Indeed, by Gårding's inequality for arbitrary $u \in \mathbb{W}_{m, 2}(\Omega)$ there are constants $c_{1}>0$ and $c_{2} \geq 0$ independent of $u$ such that

$$
(L u, u) \geq c_{1}\|u\|_{W_{m, 2}^{2}}^{2}-c_{2}\|u\|_{L_{2}}^{2}
$$

By Rellich's lemma, a bounded set in $\mathscr{W}_{m, 2}(\Omega)$ is compact in $L_{2}(\Omega)$ so that $\|u\|_{0,2}$ can be regarded as a compact seminorm on $W_{m, 2}(\Omega)$. The result now follows immediately by well-known arguments, see for example [5, pp. 126-127].

We now consider the operator defined by (4.8). First we note that by virtue of the growth condition (4.4) and the Sobolev imbedding theorem the functional

$$
\mathcal{F}(u)=\int_{\Omega} F\left(x, D^{\beta} u\right), \quad|\beta| \leq m-1,
$$

is defined on $\mathbb{W}_{m, 2}(\Omega)$. Since $F$ is a $C^{2}$ function, an elementary calculation shows that the Fréchet derivative of $\mathcal{F}(u), \mathcal{F}^{\prime}(u)$ exists and

$$
\left(\mathcal{F}^{\prime}(u), \eta\right)=\sum_{|a| s m-1} \int_{\Omega} f_{a}\left(x, D^{\beta} u\right) D^{a} \eta
$$

Therefore, by (4.8), $f(u)=\mathcal{F}^{\prime}(u)$ so that $f$ is a well-defined mapping from $\mathbb{W}_{m, 2}(\Omega) \rightarrow \mathbb{W}_{m, 2}(\Omega)$. Again the hypothesis (4.4) shows (after an elementary computation) that the Fréchet derivative of $\mathcal{F}^{\prime}(u)$ exists and is continuous; thus 
$\mathcal{F}=u$ is $C^{1}$. Furthermore the Sobolev-Kondrachov compactness theorem implies that $f_{a}$ maps weakly convergent sequences into strongly convergent sequences. Indeed, if $u_{n} \rightarrow u$ weakly in $W_{m, 2}(\Omega)$, we estimate

$$
\left\|f\left(u_{n}\right)-f(u)\right\|=\sup _{\|\eta\| \leq 1}\left(f\left(u_{n}\right)-f(u), \eta\right) .
$$

Thus by repeated use of Hölder's inequality (4.4) and Sobolev's imbedding theorem, as $n \rightarrow \infty,\left\|f\left(u_{n}\right)-f(u)\right\|=o(1)$. Thus the operator $f(u)$ maps weakly convergent sequences into strongly convergent sequences. Hence by (4.4), the functional $\mathcal{F}(u)$ is continuous with respect to weak convergence in $W_{m, 2}(\Omega)$. Next we show that $(L u, u) \rightarrow \infty$ as $\|u\| \rightarrow \infty$ for $u \in C_{R}$. Assuming, without loss of generality, that volume $(\Omega)=1$, Jensen's inequality and the evenness of $F(x, \xi)$ imply that, for $u \in C_{R}$,

$$
R=\int_{\Omega} F\left(x,|u|,|D u|, \ldots,\left|D^{m-1} u\right|\right) \geq F\left(x, \int_{\Omega}|u|, \ldots, \int_{\Omega}\left|D^{m-1} u\right|\right) .
$$

Thus by the growth condition (4.4'), $u \in C_{R}$ implies $\|u\|_{L_{1}} \leq\|u\|_{m-1,1} \leq g(R)$ where the function $g(R)$ is a bounded function of $R$ independent of $u$. Since Garding's inequality can be written in the form

$$
(L u, u) \geq \tilde{c}_{1}\|u\|_{m, 2}^{2}-\tilde{c}_{3}\|u\|_{L_{1}}^{2} \geq \tilde{c}_{1}\|u\|_{m, 2}^{2}-\tilde{c}_{2} g(R), \quad \tilde{c}>0 .
$$

We find that $(L u, u) \rightarrow \infty$ as $\|u\|_{m, 2} \rightarrow \infty$ for $u \in C_{R}$.

The functional $\mathfrak{F}(u)$ therefore satisfies all the hypotheses of Corollary $\mathbf{A}$ so that the equation $L u=\lambda \mathcal{F}^{\prime}(u)$ has a one-parameter family of nontrivial solutions $(u(R), \lambda(R))$ such that $\lambda(R) \neq 0$ and $\int F\left(x, D^{\beta} u(R)\right)=R$; which proves Theorem 5 .

(ii) Semilinear second order elliptic equations on compact manifolds. Let $\left(\pi^{N}, g\right)$ denote a compact differentiable manifold of dimension $N$ with Riemannian metric $g$. Suppose $\Delta$ denotes the Laplace-Beltrami equation on $(\mathbb{N}, g)$. Then we consider the existence of nontrivial solutions of the nonlinear equation

$$
\Delta u+\beta(x) u+\lambda f(x, u)=0
$$

where $\beta(x)$ is any smooth function on $\left.0 \pi^{N}, g\right)$ and $f(x, \xi)$ is a $C^{1}$ function of $(x, \xi)$ satisfying

$$
\frac{\partial f}{\partial \xi}>0, \quad|f(x, \xi)| \leq \text { const }\left\{1+|\xi|^{\sigma}\right\}, \quad \sigma<\frac{N+2}{N-2} .
$$

As an application of Theorem 2, we prove

Theorem 6. Under the above bypotbeses (4.11), (4.10) bas a one-parameter family of nontrivial solutions $(u(R), \lambda(R))$ for each $R>0$ with $\int_{M} F(x, u(R))=R$, 
where $F(x, 0)=0$ and $F_{u}(x, u)=f(x, u)$, provided $F(x, \xi)$ is a strictly convex $C^{2}$ function of $u$ for fixed $x$, and $F(x, \xi) \rightarrow \infty$ uniformly in $x$, as $\|\xi\| \rightarrow \infty$.

Proof. As in (i) above, it suffices to prove the existence of nontrivial weak solutions of (4.10), i.e., a function $u \in W_{1,2}(\pi, g)$ such that

$$
\int_{\prod}\{\nabla u \cdot \nabla \eta-\beta(x) u \eta\} d V=\lambda \int_{M} f(x, u) \eta d V
$$

for all $\eta \in C^{\infty}(\mathfrak{h}, g)$. Aga in as in (i), (4.12) can be written as an operator equation in $W_{1,2}(\pi, g)$ of the form $L u=\lambda N u$, where the operators $L$ and $N$ are defined implicitly by the formulae

$$
\begin{aligned}
& (L u, \eta)=\int_{M}\left[\nabla u \cdot \nabla_{\eta}-\beta(x) u \eta\right] d V, \\
& (N u, \eta)=\int_{M}[f(x, u) \eta] d V .
\end{aligned}
$$

As in the proof of Theorem $5, L$ is selfadjoint and bounded in $W_{1,2}(\mathcal{N}, g)$ where the inner product in $W_{1,2}(\pi, g)$ is defined to be $(u, v)=\int_{\pi}(\nabla u \cdot \nabla v+u v) d V$. Since $\beta(x)$ is bounded by a constant $K$ (say) (as $\pi$ is compact),

$$
(L u, u) \geq\|\nabla u\|_{L_{2}}^{2}-K\|u\|_{0,2}^{2} \geq\|u\|_{1,2}^{2}-(K+1)\|u\|_{0,2}^{2}
$$

Because a bounded set in $W_{1,2}(\pi, g)$ is compact in $L_{2}$, we find that, just as in Theorem $1, L$ has closed range and $\operatorname{dim} \operatorname{Ker} L<\infty$. Now we investigate the operator $\mathrm{Nu}$ defined by (4.14). Clearly as in Theorem 1 , the Kondrachov-Sobolev compactness theorem implies that $N$ maps weakly convergent sequences in $\left.W_{1,2} \mathcal{O}, g\right)$ into strongly convergent sequences. Thus the function $r(u)=\int_{Q} F(x, u)$ is weakly compact and $\pi^{\prime}(u)=N u$. In addition, one verifies immediately that $\pi$ is $C^{1}$. Consequently, an application of Theorem 1 yields Theorem 6 , since, as in (i), $(L u, u) \rightarrow \infty$ as $\|u\| \rightarrow \infty$ with $\int_{\Omega} F(x, u)=R$.

(iii) Periodic solutions of nonlinear Hamiltonian systems. If $x$ denotes a vector in $R^{N}$, we consider the periodic solutions of the system

$$
\ddot{x}+\nabla U(x)=0
$$

where $U(x)$ is a $C^{2}$ real-valued function defined on $\mathbf{R}^{N}$. By virtue of Theorems 1,3 , and 4 , we can prove

Theorem 7. The system (4.15) bas a one-parameter family of periodic solutions $x(R)$, parametrized by the mean value of $U(x)$ over a period, provided the function $U(x)$ is strictly convex and $0=U(0) \leq U(x)$.

Theorem 8. If, in addition to the bypotheses of Theorem 7, $U(x)=1 / 2 A x \cdot x$ $+O\left(|x|^{2}\right)$ for $|x|$ sufficiently small, where the eigenvalues $\left\{\lambda_{i}^{2}\right\}$ of the constant matrix $A$ are ordered such that $0<\lambda_{1}^{2} \leq \lambda_{2}^{2} \leq \cdots \leq \lambda_{N}^{2}$. Then $x(R) \rightarrow 0$ as $R \rightarrow 0$ 
and the minimal period of $x(R), \tau(R) \rightarrow 2 \pi / \lambda_{N} \cdot$

Theorem 9. If $U(x)=1 / 2 A x \cdot x+O\left(|x|^{2}\right)$ is a $C^{2}$ real-valued function for $|x|$ sufficiently small, where $A$ is a nonsingular matrix with $k$ positive eigenvalues; $0<\lambda_{1}^{2} \leq \lambda_{2}^{2} \leq \lambda_{3}^{2} \leq \cdots \leq \lambda_{k}^{2}$. Then in a small neighborbood of $x=0$, (4.15) bas $k$ one-parameter families of periodic solutions $x_{i}(R)(i=1, \cdots, k)$ whose periods $\tau_{i}(R)$ for $R$ sufficiently small satisfy $\left(x_{i}(R), \tau_{i}(R)\right) \rightarrow\left(0,2 \pi / \lambda_{i}\right)$ as $R \rightarrow 0$.

Proofs. We first show that the nontrivial periodic solutions of (4.15) are in (1-1) correspondence with the nontrivial solutions of an operator equation of the form (0.1). To this end, we follow the procedure in [1], and set $t=\lambda s$ in (4.15) (so that solutions of period $2 \pi$ in $s$ correspond to $2 \pi \lambda$-periodic solutions in $t$ ). Then we let $H=\Pi_{i=1}^{N} H_{i}[0, \pi]$ denote the direct product of $N$-copies of the Hilbert space $H_{i}$ of even absolutely continuous functions $x_{i}(s)$ defined on $[0, \pi]$ such that $x_{i}(s) \in L_{2}(0, \pi)(i=1, \cdots, N) . H$ is a Hilbert space with respect to the inner product

$$
(x, y)_{H}=\sum_{i=1}^{N} \int_{0}^{\pi}\left(x_{i} y_{i}+\dot{x}_{i} \dot{y}_{i}\right) d s .
$$

Following [1], even $2 \pi$-periodic solutions of $\ddot{x}+\lambda^{2} \nabla U(x)=0$ can be found by finding the nontrivial solutions of the operator equation $L x=\lambda^{2} N x$ in $H$, where the operators $L$ and $N$ mapping $H$ into $H$ are defined implicitly by the relations

$$
\begin{aligned}
& (L x \cdot \eta)_{H}=\sum_{i=1}^{N} \int_{0}^{\pi} \dot{x}_{i} \cdot \dot{\eta}_{i} \text { for all } \eta \in H, \\
& (N x \cdot \eta)_{H}=\int_{0}^{\pi} \nabla U(x) \cdot \eta .
\end{aligned}
$$

Clearly (4.16) implies that $L$ is selfadjoint, $(L x \cdot x) \geq 0, \operatorname{dim} \operatorname{Ker} L \simeq \mathrm{R}^{N}$, and that the range of $L$ is closed. On the other hand, (4.16) clearly implies that $N$ is the Fréchet derivative of $\tau^{\prime}(u)=\int_{0}^{\pi} U(x)$ in $H$, and $\tau^{\prime}(u)=\int_{0}^{\pi} U(x) d s$ is clearly weakly sequentially continuous on $H$ since bounded sets in $H$ are compact in $C[0, \pi]$. It is also clear that the strict convexity and smoothness of $U(x)$ imply the strict convexity and $C^{2}$ nature of $" \tau(u)$. Thus an application of Theorems 1 and 3 to the equation $L u=\lambda^{2} N u$ yields Theorems 7 and 8 . Indeed Theorem 8 is derived from Theorem 3 by noting that, by hypothesis, if $\nabla U(x)=A x+$ $O\left(|x|^{2}\right)$, the smallest nonzero eigenvalue of the equation $L x=\lambda L_{1} x$ corresponds to the smallest nonzero eigenvalue $\beta$ of $\ddot{x}+\lambda^{2} A x=0$ over $H$. Thus $\beta^{2}=1 / \lambda_{N}^{2}$, so that $\beta=1 / \lambda_{N}$; and thus $r(R)=2 \pi \lambda(R) \rightarrow 2 \pi / \lambda_{N}$ as $R \rightarrow 0$. In exactly the 
same way, periodic solutions of the system $\ddot{x}+A x+f(x)=0$ can be found by finding solutions of the operator equation

$$
L x=\lambda^{2}\left\{L_{1} x+P(x)\right\}
$$

in $H$. Since periodic solutions of (4.15) correspond only to strictly positive eigenvalues $\lambda^{2}$, we note that the positive eigenvalues of $L x=\lambda^{2} L_{1} x$ in $H$ correspond to positive eigenvalues of $\ddot{x}+\lambda^{2} A x=0$ in $H$. However, these last eigenvalues are precisely the numbers $\left\{N^{2} / \lambda_{i}^{2} \mid N=1,2, \ldots, i=1,2, \ldots, k\right\}$. In particular, the numbers $1 / \lambda_{i}^{2}(i=1, \ldots, ;, k)$ are eigenvalues that correspond to one-parameter families $x_{i}(R)$ of (4.15) with period $2 \pi \lambda(R) \rightarrow 2 \pi / \lambda_{i}$ as $R \rightarrow 0$.

In a similar manner Theorem 9 is derived from Theorem 4 . The only point that does not follow immediately is that the hypothesis, $L_{1}: \operatorname{Ker} L \rightarrow \operatorname{Ker} L$ is $(1-1)$, is satisfied in the present case. To verify this, we note first that $\operatorname{Ker} L \cong \mathbf{R}^{N}$ and consists of the constant $N$-vectors $\left(c_{1}, c_{2}, \cdots, c_{N}\right)$. Now on $\operatorname{Ker} L$, the abstract operator $L_{1}$ coincides with matrix multiplication by the matrix $A$. Thus since $A$ is nonsingular, $A$ is $(1-1)$ on $\operatorname{Ker} L$ and maps $\operatorname{Ker} L$ into itself.

Remark. Dr. Alan Weinstein has recently obtained an alternate proof. of Theorem 9 showing that each family of periodic solutions can be chosen to be distinct.

\section{BIBLIOGRA PHY}

1. M. S. Berger, Periodic solutions of second order dynamical systems and isoperimetric variational problems, Amer. J. Math. 93 (1971), 1-10. MR 43 \#2588.

2. An eigenvalue problem for nonlinear elliptic partial differential equations, Trans. Amer. Math. Soc. 120 (1965), 145-184. MR 31 \#6047.

3. R. T. Rockafellar, Convex analysis, Princeton Math. Series, no. 28, Princeton Univ. Press, Princeton, N. J., 1970. MR 43 \#445.

4. M. A. Krasnosel'skiY, Topological methods in the theory of nonlinear integral equations, GITTL, Moscow, 1956; English transl., Macmillan, New York, 1964. MR 20 \#3464; MR 28 \#2414.

5. M. Schechter, Principlies of functional analysis, Academic Press, New York, 1971.

6. H. Poincaré, Sur les lignes geodesique des surfaces convexes, Trans. Amer. Math. Soc. 6 (1905), 237-274.

7. S. I. Pohožaev, The set of critical values of a functional, Mat. Sb. 75 (117) (1968), 106-111 = Math. USSR Sb. 4 (1968), 93-98. MR 36 \#4586.

8. M. Morse, Calculus of variations in the large, Amer. Math. Soc. Colloq. Publ., vol. 18, Amer. Math. Soc., Providence, R. I., 1934.

9. M. Berger, Bifurcation theory and the type numbers of Marston Morse, Proc. Nat. Acad. Sci. U. S. A. 69 (1972), 1737-1738.

DEPARTMENT OF MATHEMATICS, YESHIVA UNIVERSITY, NEW YORK, NEW YORK 10033

SCHOOL OF MATHEMATICS, INSTITUTE FOR ADVANCED STUDY, PRINCETON, NEW JERSEY 08540 